\title{
Exact Splitting for Rossby Waves in a Shallow-water Model
}

\author{
By D.K. Purnell \\ New Zealand Meteorological Service \\ (Manuscript received 31 October 1986, in revised form 13 February 1987)
}

\begin{abstract}
The time-tendency operator for a shallow-water model is split into 'advection' and 'adaption' operators. Rossby eigen-modes and a gravity-wave subspace are defined for perturbations on any state where, over the whole domain, either the divergence is uniform, or else the absolute vorticity has a uniform sign. It is then shown that

a) the adaption operator yields zero tendency for Rossby modes,

b) advection does not transform gravity-wave modes into Rossby modes,

c) that (a) and (b) allow an 'exact splitting for Rossby modes'.

This splitting allows selective approximation of gravity wave modes without introducing error in the Rossby-modes, so that economical long timesteps can be used without degrading the accuracy of the part of the solution which is significant for weather prediction.

A particular computational method using this splitting is constructed and tested experimentally. This has less time-differencing error for Rossby-modes than the 'leapfrog' scheme, and has no computational mode. This particular scheme strongly attenuates high-frequency gravity wave modes.
\end{abstract}

\section{Introduction}

Numerical models with a high spatial resolution are costly. The popular 'semiimplicit' method yields economies by exploiting the circumstance that gravity wave modes of high phase speeds are of little meteorological interest. Long timesteps can be used, resulting in phase distortion of fast waves, but with little effect on slower meteorologically significant waves. In some of these schemes there is distortion of the Rossby modes but it is relatively small for two reasons. First, because the Rossby modes have lower phase speeds. Second, because the advection terms in the tendency equation which dominate the Rossby modes are not treated implicitly, so that there is some (imperfect) discrimination between Rossby modes and gravity wave modes. An exact splitting scheme can extend this strategy by exploiting perfect discrimination between Rossby modes and gravity wave modes. A similar advantage should be possible in any scheme which separates the rotational and irrotational wind tendencies, e.g. Staniforth and Temperton (1986), scheme B. Unlike that scheme, the subject to be considered is not 'leapfrog' (2-time-level) integration schemes, but 1-time-level schemes constructed with splitting approximations.

The splitting method as originally proposed was based on the observation that additive splitting of linear operators does not introduce any error provided that the operators mutually commute (Douglas and Gunn 1964, Strang 1968, Marchuk 1982 page 229, LeVeque and Oliger 1983). This principle has, however, never been directly applied to meteorological models because of the difficulty of finding a splitting where the operators do commute, and because the problem is nonlinear. The present method is based on an easier problem of finding operators that commute only on the subspace spanned by the Rossby-modes. The idea is that what 
happens outside this subspace is not so important, given that the high-frequency gravity waves can be set to zero without significant loss of meteorological information. Gravity waves of sufficiently low frequency will still be accurately represented because the splitting is at least first-order accurate in time for any mode.

The splitting of advection and adaption is chosen so that the adaption tendency operator maps every Rossby-mode to zero, and every gravity-wave mode to itself. The advection operator can be thought of as an interpolation operator which is itself a function of the trajectories. Given the trajectories this interpolation is linear and has the property that it maps the subspace of gravity-wave modes into itself; i.e., it does not transform gravitywave modes into Rossby-modes. These properties are sufficient to ensure that the splitting does not cause any error in the Rossby-modes.

The particular computational scheme to be reported here is a realization of the splitting method which gives high-fidelity approximation of Rossby-modes at very long timesteps, while also severely attenuating highfrequency gravity waves. This may have the advantage of simulating the 'geostrophic adjustment' process.

Computational economy is achieved in two ways. First, by the use of very long timesteps. Second, by the use of an inexpensive time- and space-difference approximation of the 'adaption' operator.

Since the correct adaption tendency of Rossby-modes is exactly zero, any numerical approximation of the adaption operator which is exact for stationary solutions will not contaminate the Rossby-modes. In order to ensure this, it is sufficient to use a single estimate of divergence to update both geopotential and vorticity. This simple requirement can be satisfied even when using inexpensive space-differencing approximations. The particular method used for the adaption operator is an efficient first-order accurate, frequencydamping version of the alternating-direction (ADI) approximation referred to in Marchuk (1982, p 229) as the 'stabilization method' (see also Beam and Warming 1976, page 90), which requires solution of only 1-dimensional, tridiagonal linear equations. It is also necessary to solve a 2-dimensional Poisson equation in order to separate rotational and irrotational components of wind tendency.

The advection operator was implemented by the upstream spline interpolation scheme of Purnell (1976). For long timesteps it has been found cost-effective to iterate this interpolation several times per timestep. The trajectories are determined just once per timestep, but the advection is computed as the $N$ th power of an operator interpolating upstream a fraction $1 / N$ of the full trajectory, where $N=4$ was used in the experiments. The result is equivalent to the use of curved trajectories.

\section{Splitting of the shallow-water model}

The shallow-water equations can be written

$$
\begin{aligned}
& \partial \phi / \partial t=-\phi \nabla \cdot V-V \cdot \nabla \phi \\
& \partial V / \partial t=-\eta k \times V-\nabla(\phi+K(V))
\end{aligned}
$$

for time $t$, rate of change $\partial / \partial t$, geopotential $\phi$, wind vector $V$, horizontal gradient operator $\nabla$, upward normal $k$, relative vorticity $\xi=$ $k \cdot \nabla \times V$, Coriolis parameter $f$, absolute vorticity $\eta=f+\xi$, kinetic energy $K(V)=(V \cdot V) / 2$. To avoid confusion the abbreviation $\nabla^{2}=\nabla \cdot \nabla$ will be avoided. A pure divergent component of the wind tendency is defined;

$$
\partial V_{d} / \partial t=-\nabla(\phi+K(V)+H(V))
$$

where $H(V)$ is defined;

$$
\begin{aligned}
\nabla \cdot \nabla H(V) & =\nabla \cdot(\eta k \times V) & & \text { in interior, } \\
\phi+K(V)+H(V) & =0 & & \text { on boundary. }
\end{aligned}
$$

This boundary condition is the one suggested by Bijlsma, Hafkenscheid, and Lynch (1986) for partioning the wind into irrotational and rotational components on a limited domain. Some condition at the boundary is necessary to ensure a unique partitioning, and this one has minimum divergent kinetic energy. The choice of boundary condition is irrelevant to the exactness of the splitting, but could affect the accuracy of the computation of 
trajectories. Set $V=V_{r}+V_{d}$, where $V_{r}$ is the pure rotational component

$$
\partial V_{r} / \partial t=\nabla H(V)-\eta k \times V
$$

which is equivalent to

$$
\partial \eta / \partial t=-\nabla \cdot(\eta V)
$$

So (2.1) can be rewritten

$$
\begin{aligned}
\partial \phi / \partial t & =-\phi \nabla \cdot V \quad-\quad V \cdot \nabla \phi \\
\partial \eta / \partial t & =-\eta \nabla \cdot V \quad-\quad V \cdot \nabla \eta \\
\partial V_{d} / \partial t & =-\nabla(\phi+K(V)+H(V))
\end{aligned}
$$

or

$$
\partial / \partial t\left[\begin{array}{c}
\phi \\
\eta \\
V_{d}
\end{array}\right]=A\left[\begin{array}{c}
\phi \\
\eta \\
V_{d}
\end{array}\right]+B_{(V)}\left[\begin{array}{c}
\phi \\
\eta \\
V_{d}
\end{array}\right]
$$

where the first term (operator $A$ ) is the 'adaption' part of the splitting, and the second term (linear operator $\left.B_{(V)}\right)$ is the 'advection' part, Operator $A$ specifies zero tendency for any Rossby-mode. The computational strategy is to simplify the problem by splitting, approximate $B$ well, and economise on the approximation of $A$ while keeping Rossby tendencies zero. This is ensured simply by using the same estimate of divergence for both the geopotential and vorticity tendency. The model variables are just the geopotential and the total wind; only the tendencies of wind are decomposed into rotational and irrotational parts.

\section{Eigenvectors of the adaption operator}

The Rossby-modes are often defined (e.g. Haltiner and Williams, 1980, page 40) as a particular subset of eigenvectors of the prediction equations for the simplified case where the time-tendency operator is linearized about a state of constant or zero wind. However, a more general linearization about an arbitrary state can be considered. In order to ensure the existence of Rossby eigen-modes the only cases to be considered are those where, over the whole domain, either divergence is uniform, or else the absolute vorticity has a uniform sign. These Rossby eigen-modes are here defined as a particular subset of the eigenvectors of the derivative of the adaption operator $A$ in
(2.6). These general modes are directly related to the potential-vorticity $\eta / \phi$, so they represent the waves originally identified by Rossby (1980). The adaption operator $A$ specifies zero change in $\eta / \phi$ and therefore zero change in the Rossby-modes. Another significant feature of (2.6) to be demonstrated is that the advection operator $B_{(V)}$ does not transform vectors which belong to a gravitywave subspace into Rossby modes.

The derivative of an operator $T: \mathcal{V} \rightarrow \mathscr{W}$ at the state $s \in \mathcal{Q}$ will be denoted $D_{s} T$. Define the divergence $\sigma=\nabla \cdot V=\nabla \cdot V_{d}$. Redefine the operators $A, B, K, H$ as functions of $\phi, \eta, \sigma$ instead of $\phi, \eta, V_{d}$. Then the adaption component of the tendency, part $A$ of $(2.6)$ is

$$
\partial s / \partial t=A s, \quad s=\left[\begin{array}{l}
\phi \\
\eta \\
\sigma
\end{array}\right]
$$

and near the state $\phi, \eta, \sigma$ (3.1) has the linear approximation

$$
\partial / \partial t\left[\begin{array}{l}
d \phi \\
d \eta \\
d \sigma
\end{array}\right]=-\left[\begin{array}{lll}
\sigma & 0 & \phi \\
0 & \sigma & \eta \\
\nabla \cdot \nabla & N & M
\end{array}\right]\left[\begin{array}{l}
d \phi \\
d \eta \\
d \sigma
\end{array}\right]
$$

where $d \phi, d \eta, d \sigma$ are small departures from the reference state $\phi, \eta, \sigma$, and where $N, M$ are the operators;

$$
\begin{aligned}
N= & D_{\eta}(\nabla \cdot \nabla[K+H])=\nabla \cdot \nabla\left[V \cdot k \times \nabla(\nabla \cdot \nabla)^{-1}\right] \\
& -[\nabla \eta] \cdot\left[\nabla(\nabla \cdot \nabla)^{-1}\right]-\eta-\xi \\
M= & D_{\sigma}(\nabla \cdot \nabla[K+H])=\nabla \cdot \nabla\left[V \cdot \nabla(\nabla \cdot \nabla)^{-1}\right] \\
& +[\nabla \eta] \cdot\left[k \times \nabla(\nabla \cdot \nabla)^{-1}\right]
\end{aligned}
$$

The derivative of the operator $A$ is therefore;

$$
D_{\phi, \eta, \sigma} A=-\left[\begin{array}{lll}
\sigma & 0 & \phi \\
0 & \sigma & \eta \\
\nabla \cdot \nabla & N & M
\end{array}\right]
$$

The significance of $D_{s} A$ for the dynamics of (3.1) follows from the identity

$$
\partial^{2} s / \partial t^{2}=D_{s} A[\partial s / \partial t]
$$

so that the eigenvalues of $D_{s} A$ correspond to frequencies of any wave-like solutions of (3.1). Just as in the special case where the reference state $\phi, \eta, \sigma$ is a state of uniform constant wind, $D_{\phi, \eta, \sigma} A$ can be represented on a product of two subspaces, such that 
each subspace is mapped into itself. One subspace contains Rossby-wave eigen-modes, and the other contains gravity-wave modes. On the Rossby subspace $D_{\phi, \eta, \sigma} A$ is represented by a basis of one-dimensional operators, i.e. eigenvalues $\omega$ and eigenvectors $h, q, s$ such that

$$
D_{\phi, \eta, \sigma} A\left[\begin{array}{l}
h \\
q \\
s
\end{array}\right]=\omega\left[\begin{array}{l}
h \\
q \\
s
\end{array}\right]
$$

so that

$$
\begin{aligned}
& (\sigma+\omega) h+\phi s=0 \\
& (\sigma+\omega) q+\eta s=0 \\
& \nabla \cdot \nabla[h]+N[q]+(M+\omega)[s]=0
\end{aligned}
$$

By inspection, one solution for $\omega, h, q, s$ satisfying (3.3), is,

$$
\begin{array}{r}
{\left[\begin{array}{l}
h \\
q \\
s
\end{array}\right]=\left[\begin{array}{c}
-(\nabla \cdot \nabla)^{-1} N r \\
r \\
0
\end{array}\right], \quad \omega=-\sigma,} \\
(\sigma \text { constant })
\end{array}
$$

where $r$ is an arbitrary field. This solution reduces to the usual set of Rossby-modes in the case where $\phi, \eta, \sigma$ is a state of zero wind and the Coriolis parameter is a constant $f=f_{0}$ say. In particular the coefficients of fields $r=-\exp [i(m x+n y)]$, with integers $m, n$ not both zero, in (3.4) are

$$
\left[\begin{array}{l}
h(m, n) \\
q(m, n) \\
s(m, n)
\end{array}\right]=\left[\begin{array}{c}
f_{0} /\left(m^{2}+n^{2}\right) \\
-1 \\
0
\end{array}\right]
$$

since $N=-\eta=-f_{0}$ in the case of zero wind. This is exactly the same as in Bourke and McGregor (1983).

If (3.3) has any other solutions $\omega=\lambda_{j}$, $j=1,2$, they satisfy

$$
\left[\begin{array}{l}
h \\
q \\
s
\end{array}\right]=\left[\begin{array}{c}
\phi \alpha \\
\eta \alpha \\
-\left(\sigma+\lambda_{j}\right) \alpha
\end{array}\right]
$$

where

$$
\left(M+\lambda_{j}\right)\left[\left(\sigma+\lambda_{j}\right) \alpha\right]=\nabla \cdot \nabla[\phi \alpha]+N[\eta \alpha]
$$

and $\alpha\left(\lambda_{j}\right)$ is any field that satisfies (3.6) for some scalar $\lambda_{j}$. Each solution $\alpha\left(\lambda_{j}\right)$ corresponds to one eigenvector (3.5) which belongs to gravity-wave subspace $j$. In the case where the reference state is one of uniform wind $V$, a complete set of eigenvectors exists. However, the existence of such eigenvectors is not essential to the present purpose. Since (3.4) defines the Rossby modes, it is sufficient to identify a complementary subspace which is invariant, i.e. mapped into itself under the operator $D_{s} A$, and which contains all eigenvectors (3.5). Vectors in this subspace will be called 'gravity-wave modes'. A suitable subspace is spanned by the vectors

$$
\left[\begin{array}{l}
h \\
q \\
s
\end{array}\right]=\left[\begin{array}{c}
\phi \alpha \\
\eta \alpha \\
\beta
\end{array}\right]
$$

where $\alpha$ and $\beta$ are arbitrary fields. The eigenvectors (3.5) obviously belong to the subspace (3.7), while the Rossby eigenvectors (3.4) do not belong to it and

$$
\begin{aligned}
& {\left[\begin{array}{lll}
\sigma & 0 & \phi \\
0 & \sigma & \eta \\
\nabla \cdot \nabla & N & M
\end{array}\right]\left[\begin{array}{c}
\phi \alpha \\
\eta \alpha \\
\beta
\end{array}\right]} \\
& \quad=\left[\begin{array}{l}
\phi(\sigma \alpha+\beta) \\
\eta(\sigma \alpha+\beta) \\
\nabla \cdot \nabla(\phi \alpha)+N(\eta \alpha)+M \beta
\end{array}\right]
\end{aligned}
$$

also belongs to the same subspace (3.7). Note that this subspace is invariant under $D_{s} A$ even if $\sigma$ is not a constant. It is now possible to identify that portion of any perturbation which lies in the Rossby-mode subspace. If a perturbation $d \phi, d \eta, d \sigma$ is represented as the sum

$$
\left[\begin{array}{l}
d \phi \\
d \eta \\
d \sigma
\end{array}\right]=\left[\begin{array}{c}
-(\nabla \cdot \nabla)^{-1} N r \\
r \\
0
\end{array}\right]+\left[\begin{array}{c}
\phi \alpha \\
\eta \alpha \\
\beta
\end{array}\right]
$$

with coefficient fields $r, \alpha, \beta$; then, solving for $\gamma$ and eliminating $\alpha$ and $\beta$,

$$
\begin{aligned}
{\left[\phi+\eta(\nabla \cdot \nabla)^{-1} N\right] r } & =\phi(d \eta)-\eta(d \phi) \\
& =\phi^{2} d(\eta / \phi)
\end{aligned}
$$

so the amplitude of the Rossby-mode $=r$, which is proportional to the perturbation of potential vorticity $\eta / \phi$. It immediately follows that the adaption operator $A$ specifies zero tendency for the Rossby-modes, as claimed, since the adaption tendency is 
$\partial r / \partial t$

$$
\begin{aligned}
& =\left[\phi+\eta(\nabla \cdot \nabla)^{-1} N\right]^{-1}[\phi(\partial \eta / \partial t)-\eta(\partial \phi / \partial t)] \\
& =0 \quad \text { from (3.1) and (2.5), (2.6), }
\end{aligned}
$$

provided the operator $\left[\phi+\eta(\nabla \cdot \nabla)^{-1} N\right]$ is nonsingular.

In the case where the absolute vorticity is nonzero with a uniform sign over the whole domain, the operators $A, B, K, H, N, M$ can be re-defined as maps on the variables $\log (\phi)$, $\log |\eta|, \sigma$ instead of $\phi, \eta, \sigma$. Then

$$
D_{s} A=\left[\begin{array}{ccc}
0 & 0 & 1 \\
0 & 0 & 1 \\
\nabla \cdot \nabla & N & M
\end{array}\right]
$$

and the Rossby modes are the same as before (3.4), with the eigenvalue $\omega=0$. The gravitywave subspace remains invariant as already noted. The basis for this subspace corresponding to (3.7) for the transformed variables $\log (\phi), \log |\eta|, \sigma$ is

$$
\left[\begin{array}{l}
d(\log (\phi)) \\
d(\log |\eta|) \\
d \sigma
\end{array}\right]=\left[\begin{array}{l}
\alpha \\
\alpha \\
\beta
\end{array}\right]
$$

for arbitrary fields $\alpha, \beta$.

Another interesting and significant property of the solutions (3.4), (3.5), (3.6) is the response of the gravity-wave modes to the advection operator $B_{(V)}$ of (2.6). Integration of advection for a time $t$ is equivalent to upstream interpolation, which can be represented as a linear operator $Q_{t}$, itself a function of the velocities $V$ over the whole time interval 0 to $t$. The effect of $Q_{t}$ on any vector in the gravity-wave subspace (3.7) is

$$
Q_{t}\left[\begin{array}{r}
\phi(0) \alpha(0) \\
\eta(0) \alpha(0) \\
\beta(0)
\end{array}\right]=\left[\begin{array}{r}
\phi(t) \alpha(t) \\
\eta(t) \alpha(t) \\
\beta(t)
\end{array}\right]
$$

where

$$
\begin{aligned}
& \phi(t) \text { is the upstream value of } \phi(0) \\
& \eta(t) \text { is the upstream value of } \eta(0) \\
& \alpha(t) \text { is the upstream value of } \alpha(0) \\
& \beta(t)=\beta(0)
\end{aligned}
$$

This vector (3.10) also belongs to the gravitywave subspace (3.7), so the advection operator $Q_{t}$ does not produce any Rossby-wave com- ponent from the original gravity-wave mode.

In circumstances where there exists a complete set of gravity-wave eigenvectors (3.5) the effect of the operator $Q_{t}$ on a gravitywave mode belonging to subspace 1 will be to split it into a combination of gravity-wave modes, one set of modes belonging to the original subspace 1 , and the other set belonging to the subspace 2 . The eigen-frequencies $\lambda_{1}, \lambda_{2}$ of (3.6) are complex conjugutes which correspond to waves propagating in opposite directions. So it seems reasonable to interpret this effect as a reflection/refraction/ distortion of gravity waves.

\section{A splitting which is exact for Rossby waves}

The idea of splitting as a numerical method for computing the time-evolution of a state $s$ which according to

$$
\partial s / \partial t=A[s]+B_{(V)}[s]
$$

is to first construct a solution operator $P_{t}$ for the sub-problem

$$
\partial p / \partial t=A[p]
$$

with the solution;

$$
p(t)=P_{t}[p(0)]
$$

and to construct a solution operator $Q_{t}$ for the sub-problem

$$
\partial q / \partial t=B_{(V)}[q]
$$

with the solution;

$$
q(t)=Q_{t}[q(0)]
$$

Then if $A B_{(V)}=B_{(V)} A$ the original problem (4.1) has the solution

$$
s(t)=P_{t} Q_{t}[s(0)]
$$

(Douglas and Gunn 1964, Marchuk 1982). Otherwise, if $A$ and $B_{(V)}$ do not commute, neither do $P_{t}$ and $Q_{t}$, and the solution (4.6) is only approximate. Approximations of this sort may be good enough for some purposes though. If (4.3) and (4.5) approximate (4.2) and (4.4) with second-order accuracy in time, then the approximation of (4.1) due to Strang (1968) 


$$
s(2 t)=Q_{t} P_{t} P_{t} Q_{t}[s(0)]
$$

is second order accurate in time also, although the splitting introduces some error. The size of this error depends on how nearly $A$ commutes with $B_{(v)}$ (Strang 1968).

However, the goal here is a splitting which is 'exact in the Rossby-mode subspace'. Define $R$ to be the projection onto the Rossbymode subspace, i.e. for any Rossby-mode $r$, $r=R r$, and for any state $g$ which has no Rossby-mode component, $R g=0$. Then the numerical solution (4.6) will be called 'exact for Rossby waves' if

$$
R s(t)=R P_{t} Q_{t}[s(0)]
$$

where $s(t)$ is the true solution at time $t$, and $R s(t)$ is its Rossby-mode component. In other words, the right-side of (4.8) evaluates to the correct Rossby-mode component.

Since the operator $A$ in (3.1) maps Rossbymodes to zero (3.9) it follows that

$$
P_{t} r=r
$$

is exact for any Rossby-mode $r$. Also since the operator $A$ maps gravity-wave modes to themselves, so does $P_{t}$, so that

$$
R P_{t} Q_{t}=R Q_{t}
$$

Therefore, if (4.5) is exact, and (4.9) is fulfilled, it follows that (4.8) is exact. This proves that the splitting (4.1)-(4.6) is 'exact for Rossby waves', where the operators $A$ and $B$ are defined as in (2.5), (2.6). As already noted, (4.9) is a very easy requirement to fulfil. There are very many possibilities for numerical approximation of the 'adaption' operator $A$, subject to the simple restriction that the same estimate of the divergence $\nabla \cdot V$ must be used to update both $\phi$ and $\eta$.

The practical numerical integration scheme works by repetition of (4.6) for small timesteps $t$. This in fact requires a stronger condition than (4.8) for individual steps in order that (4.8) be satisfied at time $n t$ for integers $n>1$. It is sufficient that

$$
R s(n t)=R P_{t} Q_{t}[R s((n-1) t)]
$$

This stronger condition (4.10) is also satisfied by the splitting (4.1)-(4.6). The dotted arrows in the schematic diagram below represent transformations which would cause contamination of the Rossby modes at timesteps following the first step.
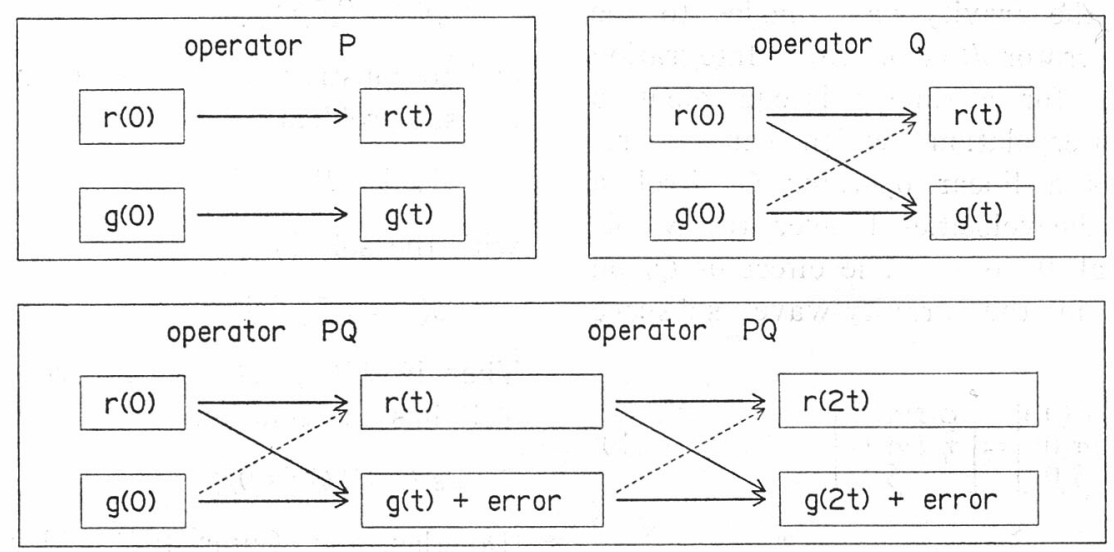

However, $R P_{t} g=0$ for any gravity mode $g$, and the operator $Q_{t}$ does not transform gravity-waves into Rossby-waves (para. 4), so the dotted-arrow transformation does not exist. Hence

$$
\begin{aligned}
R P_{t} Q_{t} s & =R P_{t} R Q_{t} s \\
& =R P_{t} Q_{t} R s
\end{aligned}
$$

so that (4.8) then implies (4.10).

The practical advantage of a splitting which is 'exact for Rossby waves' is that the method can economize on the gravitywave solution, yet be accurate for very long timesteps (see para. 5), since the Rossby waves are the meteorologically important component of the solution. 


\section{Approximation of adaption to allow long timesteps}

The time-difference scheme used to construct the operator $P_{t}$ (4.3) was a first-order accurate, frequency-damping version of the alternating-direction (ADI) approximation referred to in Marchuk (1982, p 226) as the 'stabilization method' (see also Beam and Warming 1976, page 90), which requires solution of only 1-dimensional, tri-diagonal linear equations. The scheme can be written

$$
X Y\left(p^{t+\tau}-p^{t}\right)=\tau A p^{t}, \quad p=\left[\begin{array}{l}
\phi \\
\eta \\
u \\
v
\end{array}\right]
$$

where $u, v$ are components of the wind $V$ in the $x$ and $y$ directions, and

$$
\begin{aligned}
& X=\left[\begin{array}{cccc}
1 & 0 & \varepsilon \phi \partial / \partial x & 0 \\
0 & 1 & 0 & 0 \\
\varepsilon \partial / \partial x & 0 & 1 & 0 \\
0 & 0 & 0 & 1
\end{array}\right] \\
& Y=\left[\begin{array}{cccc}
1 & 0 & 0 & \varepsilon \phi \partial / \partial y \\
0 & 1 & 0 & 0 \\
0 & 0 & 1 & 0 \\
\varepsilon \partial / \partial y & 0 & 0 & 1
\end{array}\right]
\end{aligned}
$$

The ADI method referenced above is secondorder accurate and unconditionally stable with $\varepsilon=\tau / 2$, however this does not guarantee the stability of the entire scheme (4.6). Experiments indicate that the scheme is not stable with $\varepsilon=\tau / 2$, whereas no instability has ever been observed when using the first-order accurate, frequency-damping version, given by $\varepsilon=\tau$. Some clarification of the need for dissipation of this sort may be inferred from Kreiss theory of dissipative difference schemes (see e.g. Richtmyer and Morton 1967, chapter 5.4) and from Robert, Shuman and Gerrity (1970).

Spatial approximations for the operator $P_{t}$ were of the conventional centred-difference type on the Arakawa 'C' grid (see e.g. Haltiner and Williams 1980, page 226)

\section{Second-order accurate time differencing for Rossby modes}

A second-order-accurate advection method is developed first for the case where a firstorder-accurate estimate of the trajectories is available. A complete second-order-accurate scheme is then achieved with a timewise staggering of the divergent component of the wind and a correction of a preliminary estimate of the rotational component of trajectories.

Consider 1-dimensional advection of $\phi$ by wind $u$ at location $x$ and time $t$;

$$
\partial \phi(x, t) / \partial t=-u(x, t) \partial \phi(x, t) / \partial x
$$

or $\partial \phi / \partial s=0$, on the characteristic curve $\partial(x-t u) / \partial s=0$.

Let $\phi_{t}=\partial \phi / \partial t, \phi_{x}=\partial \phi / \partial x$.

Since $\phi$ is conserved along the characteristic, a natural computational method for $\phi$ at the point $x=0, t=2 \tau$, is the upstream interpolation estimate

$$
\phi^{*}(2 \tau)=\phi\left(-2 \tau u^{*}(\tau), 0\right)
$$

where

$$
\begin{aligned}
u^{*}(\tau)= & u(-\tau u(0,0), 0)+\tau u_{t}(0,0) \\
& + \text { terms of order } \tau^{2} .
\end{aligned}
$$

is a first-order-accurate estimate of the trajectory which passes though the point $(0,2 \tau)$. Then $(6.2)$ can be shown to approximate (6.1) with second-order-accuracy in time as follows. Let $\sim$ denote equivalence up to terms of order $\tau^{3}$, and for brevity write $\phi(t)$ for $\phi(0, t)$ and $u(t)$ for $u(0, t)$, so that

$$
\begin{aligned}
u^{*}(\tau)= & u(0)-\tau u(0) u_{x}(0)+\tau u_{t}(0,0) \\
& + \text { terms of order } \tau^{2} .
\end{aligned}
$$

Note the Taylor expansion using (6.1)

$$
\begin{aligned}
\phi^{*}(2 \tau) \sim \phi(0)-2 \tau u^{*}(\tau) \phi_{x}(0)+2 \tau^{2}\left(u^{*}(\tau)\right)^{2} \phi_{x x}(0) \\
\sim \phi(0)-2 \tau u^{*}(\tau) \phi_{x}(0)+2 \tau^{2}(u(0))^{2} \phi_{x x}(0) \\
\sim \phi(0)-2 \tau u^{*}(\tau) \phi_{x}(0) \\
\quad-2 \tau^{2}\left[u(0) u_{x}(0) \phi_{x}(0)+u(0) \phi_{x t}(0)\right]
\end{aligned}
$$

also, 


$$
\begin{aligned}
\phi(2 \tau) \sim & \phi(0)-2 \tau u(0) \phi_{x}(0)-2 \tau^{2} u_{t}(0) \phi_{x}(0) \\
& -2 \tau^{2} u(0) \phi_{x t}(0)
\end{aligned}
$$

The error in the approximation (6.2) is therefore

$$
\begin{aligned}
& \phi^{*}(2 \tau)-\phi(2 \tau) \\
& \sim 2 \tau\left[u(0)-u^{*}(\tau)\right] \phi_{x}(0) \\
&+2 \tau^{2}\left[-u(0) u_{x}(0) \phi_{x}(0)+u_{t}(\tau) \phi_{x}(\tau)\right] \\
& \sim 2 \tau\left[\tau u(0) u_{x}(0)-\tau u_{t}(0)\right] \phi_{x}(0) \\
& \quad+2 \tau^{2}\left[-u(0) u_{x}(0) \phi_{x}(0)+u_{t}(\tau) \phi_{x}(\tau)\right]=0 .
\end{aligned}
$$

So the accuracy is second-order as claimed.

For multi-dimensional advection of $\phi(x, t)$ at position vector $x$, by a vector wind $V$;

$$
\partial \phi / \partial t=-V \cdot \nabla \phi
$$

a natural generalization of the estimate (6.2) for $\phi(0,2 \tau)$ is

$$
\phi^{*}(2 \tau)=\phi\left(-2 \tau V^{*}(\tau), 0\right)
$$

where

$$
\begin{aligned}
V^{*}(\tau)= & V(-\tau V(0,0), 0)+\tau V_{t}(0,0) \\
& + \text { terms of order } \tau^{2} .
\end{aligned}
$$

A similar argument shows that (6.4) approximates (6.3) with second-order accuracy. However the method employed in the experiments is the scheme of Purnell (1976) for second-order-accurate composition of the onedimensional upstream interpolation operators (6.2).

The above approximation schemes for advection both require a first-order-accurate estimate of the trajectories. The complete scheme is developed by adding an inexpensive elaboration of the computation of windtendency from vorticity-tendency. The divergent component of the wind is carried at times which are offset by half of a timestep of length $2 \tau$ as follows;

$$
\begin{aligned}
& -\cdots(3 \tau) \\
& ---\sigma(2 \tau), \xi(2 \tau) \\
& -\phi(\tau) \\
& \phi(0), \xi(0)
\end{aligned}
$$

This scheme simplifies the problem of esti- mating trajectories on the time interval $[0,2 \tau]$ to that of estimating the rotational component of these trajectories, since the divergent component of the wind at time $\tau$ is already known with sufficient accuracy. A preliminary estimate of the vorticity at $2 \tau$ is computed using the preliminary trajectories

$$
V^{* *}(\tau)=V(-\tau V(0,0), 0)
$$

giving the preliminary estimate of vorticity at time $2 \tau$;

$$
\eta^{* *}(2 \tau)=\eta\left(-2 \tau V^{* *}(\tau), 0\right)
$$

corresponding to (6.4). This estimate of $\eta^{* *}(2 \tau)$ has the error

$$
\varepsilon(2 \tau)=\eta^{* *}(2 \tau)-\eta(2 \tau) \sim-2 \tau\left[\tau V_{t}(0,)\right] \nabla \eta(0)
$$

since the term $\tau V_{t}(0,0)$ has been omitted from the trajectory. The idea is to repair this error in the vorticity-to-wind computation. The corrected vorticity is

$$
\eta^{*}(2 \tau) \sim \eta^{* *}(2 \tau)-\varepsilon(2 \tau)
$$

and a stream-function correction $\Delta \psi$ is defined:

$$
\begin{aligned}
\nabla \cdot \nabla(\Delta \psi) & =\eta^{*}(2 \tau)-\eta(0) \\
& \sim\left[\eta^{* *}(2 \tau)-\eta(0)\right]-\varepsilon(2 \tau) \\
& \sim\left[\eta^{* *}(2 \tau)-\eta(0)\right]+\tau[k \times \nabla(\Delta \psi)] \nabla \eta(0)
\end{aligned}
$$

so the stream-function correction $\Delta \psi$ can be computed by solving

$$
[\nabla \cdot \nabla-\tau \nabla(\eta(0)) \times \nabla](\Delta \psi)=\eta * *(2 \tau)-\eta(0)
$$

from which the rotational component of the wind can be stepped forward to the time $2 \tau$ with second-order-accuracy since (6.6) is second-order-accurate. Accurate trajectories can then be interpolated for the advection of $\phi$ to the time $2 \tau$.

\section{Experiments to measure accuracy as a function of timestep}

The shallow-water model was tested as a barotropic 500 hpa prediction model starting with real data on a 49 by 63 grid with a nominal spacing of $190 \mathrm{~km}$, by comparing integrations to 24 hours with one generated using 5 minute timesteps (Figs. 1, 2). For 

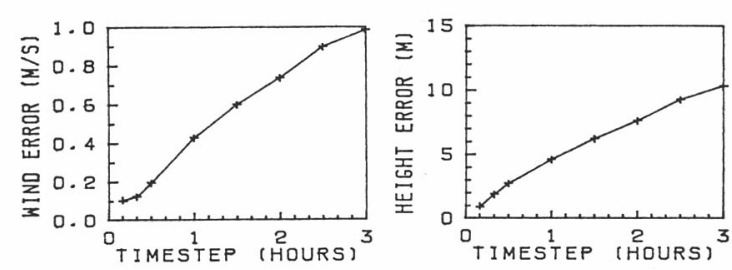

Fig. 1. RMS wind and height error as a function of timestep.

timesteps of 5 minutes up to 3 hours the RMS wind error increased from 0 up to 0.95 metres/second, which is a mean-square error of $0 \%$ up to $1 \%$ of the total mean-square difference between the initial state and the final state 24 hours later. This level of accuracy is reached if advection is calculated with several upstream interpolations per timestep, though trajectories are calculated just once per timestep. The method is numerically stable for any timestep of interest without any added spatial filtering, and no diffusion or filtering was added in the experiments. The advection method has an intrinsic spatial filtering effect (Purnell 1976), and the approximation of the adaption operator filters our high frequency gravity waves.

Figure 1a shows the RMS wind vector difference between an integration to 24 hours made with a timestep of 5 minutes and other integrations made with timesteps of from 10 minutes to 3 hours. The figure is consistent with convergence of the integrations to a limit as the timestep is reduced, as expected.

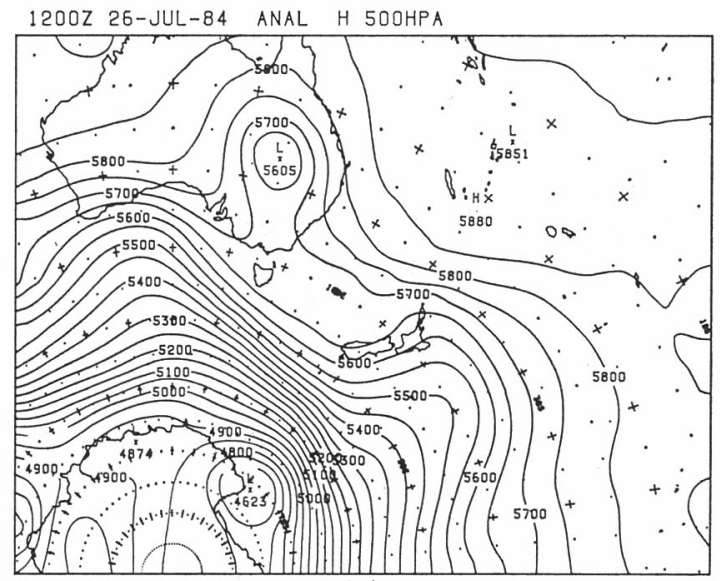

(a)

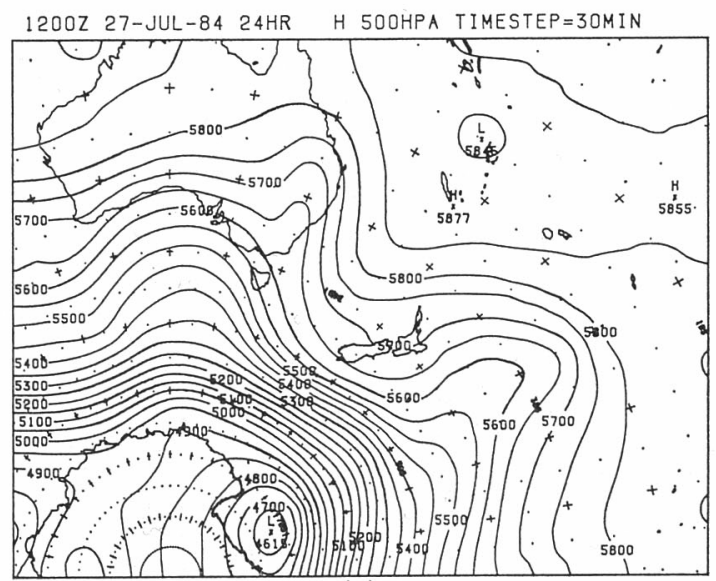

(c)

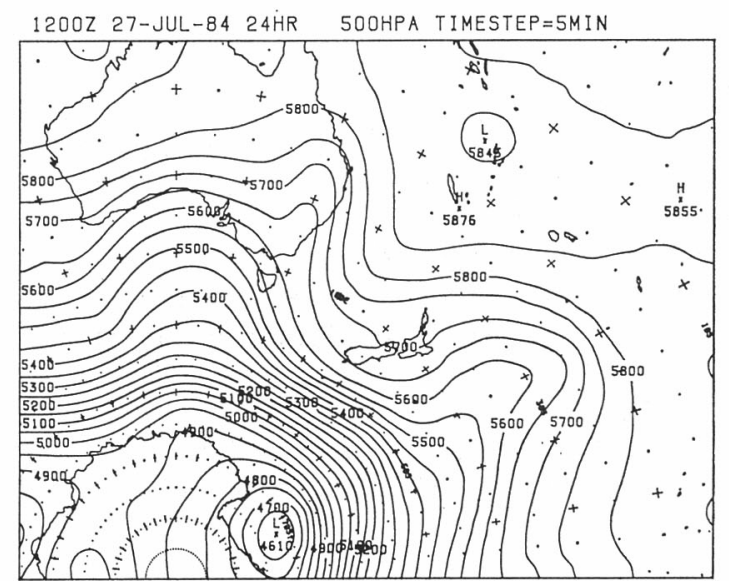

(b)

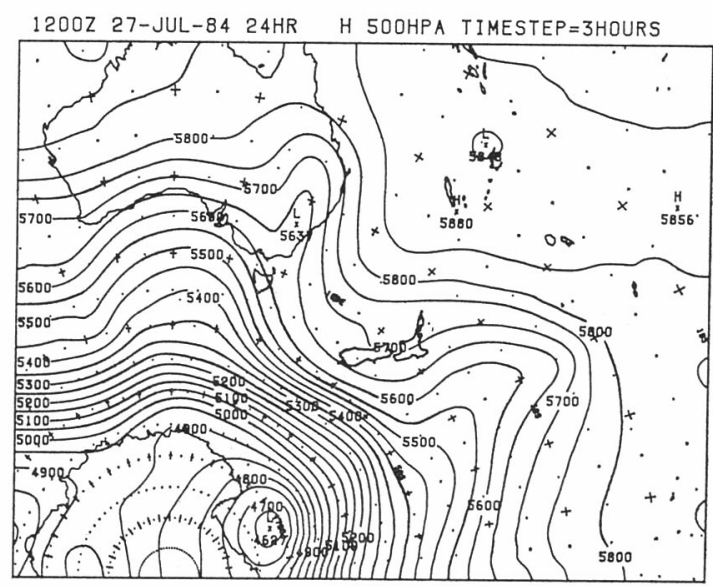

(d)

Fig. 2. Heights of initial state and 24-hour states reached with timesteps of 5, 30 and 180 minutes. 
Since the finite-difference approximations are consistent with the shallow-water differential equations, this limit must be unique and equal to the exact solution of the space-discretized time-differential shallow-water equation. The difference between this limit and an integration with a nonzero timestep is attributed to timedifferencing error. Since the integration with a 5 minute timestep is so near to this limit, the differences shown in figure 1 are very nearly equal to the time-differencing error.

Figure 1b shows corresponding RMS height differences for the same range of timesteps as in figure 1 a.

Figure 2 shows the geopotential height fields for the initial state and several of the 24 hour integrations. For the purposes of practical prediction the differences appear to be negligible for timesteps of up to 3 hours.

\section{References}

Beam, R. and R. Warming, 1976: An Implicit Finite-Difference Algorithm for Hyperbolic Systems in Conservation-Law form. J. Comput. Phys., 22, 87-110.

Bijlsma, S. J., L. M. Hafkenscheid and P. Lynch, 1986: Computation of the Streamfunction and Velocity Potential and Reconstruction of the Wind Field. Mon. Weather Rev., 114, 15471551.

Bourke, W. and J.L. McGregor, 1983: A Nonlinear Vertical Mode Initialization Scheme for a
Limited Area Prediction Model. Mon. Weather Rev., 111, 2285-2297.

Douglas, J. and J.E. Gunn, 1964: A General Formulation of Alternating Direction Methods. Numerische Math., 6, 428-453.

Haltiner, G. J. and R. T. Williams, 1980: Numerical Prediction and Dynamic Meteorology, Second ed, John Wiley.

LeVeque, R. J. and J. Oliger, 1983: Numerical Methods Based on Additive Splittings for Hyperbolic Partial Differential Equations. Math. Comp., 40, 469-497.

Marchuk, G.I., 1982: Methods of Numericol Mathematics, Second ed, Springer-Verlag.

Purnell, D. K., 1976: Solution of the Advection Equation by Upstream Interpolation with a Cubic Spline. Mon. Weather Rev., 104, 42-48.

Richtmyer, and . Morton, 1967: Difference Methods for Initial-Value Problems, Second ed, John Wiley.

Robert, A.J., F.G. Shuman and J.P. Gerrity, 1970: On Partial Difference Equations In Mathematical Physics. Mon. Weather Rev., 98, 1-6.

Rossby, C.G., 1940: Planetary flow patterns in the atmosphere. Quart. J. Roy. Meteor. Soc., 66, 68-87.

Staniforth, A. and C. Temperton, 1986: SemiImplicit Semi-Lagrangian Integration Schemes for a Barotropic Finite-element Regional Model. Mon. Weather Rev., 114, 2078-2090.

Strang, G., 1968: On the construction and comparison of difference schemes. SIAM J. Numer. Anal., 5, 506-517. 